\title{
Choroidal Thickness Changes after Orthokeratology Lens Wearing in Young Adults with Myopia
}

\author{
Jang Hun Lee ${ }^{a}$ In Hwan Hong ${ }^{b}$ Tae Yeem Lee Jae Ryong Han $^{b}$ \\ Gang Seok Jeon ${ }^{a}$ \\ aDepartment of Ophthalmology, Dasan Samsung Bright Eye Clinic, Gyeonggi-do, South Korea; ${ }^{b}$ Department of \\ Ophthalmology, Dongtan Sacred Heart Hospital, College of Medicine, Hallym University, Gyeonggi-do, South Korea; \\ 'Galmae Samsung Bright Clinic, Gyeonggi-do, South Korea
}

\section{Keywords}

Choroidal thickness · Orthokeratology · Myopia

\begin{abstract}
Introduction: Recently in South Korea, there are increasing number of young adults undergoing orthokeratology treatment for myopia control. They prefer orthokeratology treatment more than wearing spectacles or having a refractive surgery for several reasons. However, there is little research on the effect of orthokeratology treatment on choroids. $\mathbf{O b}$ jective: The aim of this study was to analyze the change in choroidal thickness (CT) in the horizontal axis in young myopic adults after orthokeratology treatment. Methods: This was a retrospective research among young myopic patients $(-1.0$ to -5.0 diopters) aged $19-29$ years $(n=36 ; 23.6 \pm 2.5$ years). We selected patients who were treated with orthokeratology for 12 months. CT values of the horizontal axis near the fovea before and after orthokeratology treatment were analyzed using optical coherence tomography. The value was measured at the beginning of treatment and at 3,6 , and 12 months after orthokeratology treatment. Three regional areas of choroid on the horizontal plane including fovea were analyzed. Result and Conclusions: In the beginning of orthokeratology treatment, CT of the horizontal axis
\end{abstract}

was $248.9 \pm 45.7 \mu \mathrm{m}$ in the temporal region, $259.9 \pm 55.3 \mu \mathrm{m}$ in the macular region, and $219.2 \pm 46.4 \mu \mathrm{m}$ in the nasal region. Three months after orthokeratology treatment, thickness values of choroids in the 3 divided areas increased significantly $(p<0.05)$. Mean CT at 6 or 12 months after orthokeratology treatment was greater than before orthokeratology treatment. CT increased after 3 months of orthokeratology treatment in each regional area. In young myopic adults, CT in nasal area was thinner than that in foveal or temporal area before treatment. CT recovered to near baseline when it was observed for more than 6 months after orthokeratology treatment.

(c) 2020 S. Karger AG, Basel

\section{Introduction}

Various methods including spectacles, contact lenses, and refractive surgeries have been developed to correct myopic refractive error. Orthokeratology contact lenses were originally fitted in the late 1960s and continued through the 1980s [1]. Since then, studies have consis-

Jang Hun Lee and In Hwan Hong contributed equally to this work and are considered co-first authors.

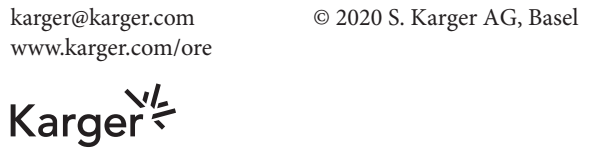


tently reported that orthokeratology treatment can significantly inhibit myopia progression in children [2-5]. It has been hypothesized that this effect may result from the induction of peripheral myopic defocus due to effects of orthokeratology lens on the mid-peripheral cornea $[5,6]$. The choroid of the eye is primarily a vascular structure supplying the outer retina [7]. In addition to its vascular functions, the choroid has been shown to change its thickness in both myopigenic and anti-myopigenic experimental conditions [8]. For example, when myopia is induced in young animals using negative lenses (imposed hyperopic defocus), the initial ocular response is a thinning of the choroid [9-11]. Conversely, if animals are reared with positive lenses (imposed myopic defocus), choroidal thickness (CT) dramatically increases within a short period in order to "push" the retina toward the new image plane to obtain a sharper image [9-11].

Recently in South Korea, many growing children have orthokeratology treatment for myopia control. Some young adults with myopia prefer orthokeratology treatment more than wearing spectacles or having refractive surgery. However, there is little research on the effect of orthokeratology treatment on choroids. Four studies in children have been reported. Gardner et al. [12] reported no significant increase in CT during a long-term followup in children in 2015. Chen et al. [13] and Li et al. [14] reported regional choroidal thickening about $21.8 \pm 25.2$ $\mu \mathrm{m}$ and $15.78-21.03 \mu \mathrm{m}$ in short period after orthokeratology treatment in children in 2016 and 2017, respectively. Most recently, Jin et al. [15] reported an increase in CT from about 8.4 to $13.5 \mu \mathrm{m}$ after orthokeratology treatment in children. However, studies about associations between orthokeratology treatment and choroid in young myopic adults have not been reported yet. Therefore, the object of this study was to investigate choroidal changes after orthokeratology treatment in young adults with myopia.

\section{Material and Methods}

We reviewed charts of 36 adult patients aged 19-29 years old who were treated with orthokeratology for myopia. Refractive error of the subject was selected based on myopia between -1.00 and -5.00 diopters $(\mathrm{D})$ and astigmatism of no more than $-1.00 \mathrm{D}$. This retrospective study included 36 patients who were followed up for 1 year after orthokeratology treatment at the Samsung Bright Eye Clinic between July 2017 and November 2018. This study was conducted in accordance with the tenets of the Declaration of Helsinki and approval from the Institutional Review Board/Ethics Committee of the Dongtan Sacred Heart Hospital (IRB No. HDT 2020-05-018). Informed consent was obtained in writing from all participants after explain the nature of orthokeratology. Manifested refraction was performed after auto-refraction. All subjects were examined by an experienced ophthalmologist for visual acuity, slit lamp microscopy, nonmydriatic fundus examination, and optical coherence tomography (OCT) for general eye health status. Patients were selected for this research if orthokeratology lens were fitted optimally for follow-up periods. Optimal fitting was determined based on fluorescein staining pattern and corneal topography as the central position of the orthokeratology lens on the cornea. All patients had a best corrected visual acuity of 0.0 LogMAR or better. They had no history of ocular or systemic disease. Noncycloplegic refraction was performed to avoid any pharmacological effect of the cycloplegic drug on CT. Basic values such as flat keratometry, steep keratometry, lens thickness, anterior chamber depth, and axial length were measured with noncontact optical biometry (IOLMaster, Zeiss Meditec, Jena, Germany).

Orthokeratology lenses in this study were rigid gas permeable materials used for CRT lenses (Paragon Vision Sciences, Mesa, AZ, USA) with the following parameters: base curve radius, $8.41 \pm 0.26$ $\mathrm{mm}$ (7.90-9.00); return zone depth, $526.79 \pm 21.66 \mu \mathrm{m}$ (500-575); and landing zone angle, $32.86 \pm 1.05^{\circ}(31-35)$. Trial lenses were derived from nomograms in the form of sliding tables produced by the manufacturer Paragon CRT sigmoid reverse geometry contact lens $[16,17]$. Patients were fitted with lenses according to the manufacturer's specifications. During the follow-up period, the orthokeratology lens were worn while sleeping at night. It was immediately removed in the morning. Examination was performed by the same ophthalmologist. Each quantitative value was measured 3 times by the same operator to minimize error of measured data.

\section{Choroid Image and Thickness Measurement}

Cross-sectional images were obtained with a spectral domain OCT instrument (Cirrus-HD OCT, Carl Zeiss Meditec, Inc., Dublin, CA, USA). To prevent measurement errors, we selected images with signal strength of 6 or higher $[15,18,19]$.

OCT examinations were performed without pupil dilation by 1 specially trained operator. CT was measured on 1 horizontal axis scan passing through the fovea. It was obtained using HD-5 Line Raster in $6 \mathrm{~mm}$ mode. CT was measured independently by 2 retina specialists using the ruler of the Zeiss OCT software. They were masked as to treatment and to each other's measurements. CT was defined as the vertical distance between the hyper-reflective line of the Bruch membrane and the hyper-reflective line of the innermost point of the choroid-scleral junction (Fig. 1) [20]. A crosssectional choroid image of about $6 \mathrm{~mm}$ of the subfovea was obtained and the central $4.2-\mathrm{mm}$ region was analyzed for consistency of data analysis, excluding optical reflectivity loss of the peripheral region. The choroid around the fovea, nasal, and temporal area within a $4.2-\mathrm{mm}$ region was selected for data analysis.

\section{Data Analysis}

SPSS 19.0 (SPSS Inc., Chicago, IL, USA) was used for all statistical analysis. Results were expressed as mean \pm standard deviation. Paired $t$ test was performed to compare CTs at initial and after 3, 6, and 12 months of orthokeratology treatment. Statistical significance was considered when $p$ value was $<0.05$. The Tukey test was chosen to compare the average CT difference between regions. To estimate reliability, the interobserver intraclass coefficients were assessed. 


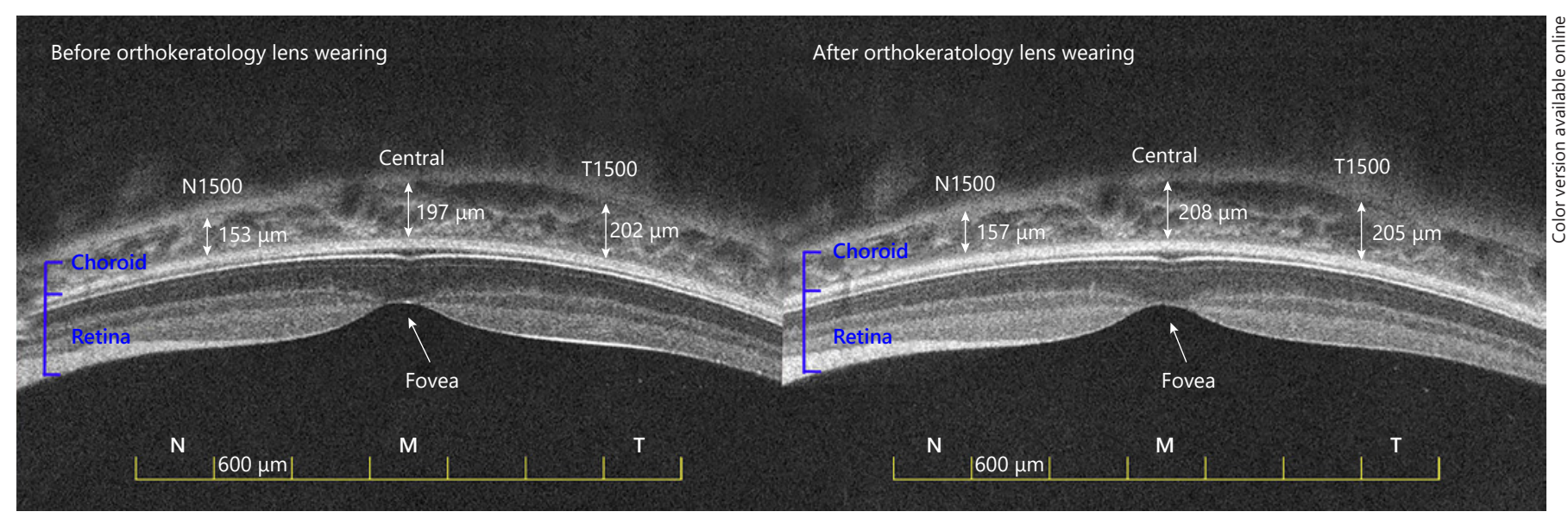

Fig. 1. Measurement of CT on a horizontal axis scan obtained using SD-OCT. An upside-down illustration of the method used to analyze CT measurement on a SD-OCT image of a subject's eye. $\mathrm{CT}$ was defined as the vertical distance between the hyper-reflec- tive line of the Bruch membrane and the innermost point of the choroid-scleral junction. SD-OCT, spectral domain optical coherence tomography; CT, choroidal thickness.

Table 1. Biometric parameters and change after overnight orthokeratology treatment

\begin{tabular}{lllrll}
\hline Time & K1, D & K2, D & LT, mm & ACD, mm & AL, mm \\
\hline Baseline & $42.75 \pm 0.89$ & $43.35 \pm 0.87$ & $3.51 \pm 0.62$ & $3.67 \pm 0.14$ & $25.51 \pm 0.98$ \\
Month 3 & $40.27 \pm 0.89$ & $41.23 \pm 0.88$ & $3.52 \pm 0.61$ & $3.64 \pm 0.45$ & $25.59 \pm 0.48$ \\
Month 6 & $40.38 \pm 0.91$ & $41.32 \pm 0.89$ & $3.51 \pm 0.14$ & $3.65 \pm 0.13$ & $25.37 \pm 0.72$ \\
Month 12 $_{p \text { value }^{\mathrm{a}}}^{40.60 \pm 0.90}$ & $41.37 \pm 0.94$ & $3.52 \pm 0.55$ & $3.66 \pm 0.19$ & $25.54 \pm 0.81$ \\
\hline
\end{tabular}

$p$ values are comparisons between baseline and after 12 months of orthokeratology treatment. K1, flat keratometry of central 3-mm zone; K2, steep keratometry of central 3-mm zone; LT, lens thickness; ACD, anterior chamber depth; AL, axial length. ${ }^{\text {a }}$ Paired $t$ test.

\section{Results}

The average sphere of patients was $-2.32 \pm 0.99 \mathrm{D}$ (range, -1.00 to $-5.00 \mathrm{D})$. Uncorrected visual acuity of all subjects during the observation period was better than 0 LogMAR after orthokeratology treatment. Initial flat keratometry and steep keratometry of central 3-mm zone were $42.75 \pm$ $0.89 \mathrm{D}$ and $43.35 \pm 0.87 \mathrm{D}$, respectively. They decreased to $40.60 \pm 0.90 \mathrm{D}$ and $41.37 \pm 0.94 \mathrm{D}$ at 12 months, respectively $(p<0.01$; Table 1$)$. Values for lens thickness, anterior chamber depth, or axial length did not change significantly during the observational period. CT before orthokeratology treatment, the initial thickness of the choroid in the horizontal axis, was the greatest at macular (M) region $(259.92 \pm 55.33 \mu \mathrm{m})$ and the thinnest at nasal $(\mathrm{N})$ region $(219.23 \pm 46.46 \mu \mathrm{m})$ (Table 2). After 3 months of orthokeratology treatment, the CT in each region of the horizon- tal axis was significantly thicker than the initial value (Table 2; Fig. 2). The greatest increase in thickness was in the $\mathrm{M}$-region while the smallest increase was in the $\mathrm{N}$-region. Figure 2 shows longitudinal change of mean CT for each region over a 12-month period. The increase in CT was found to be closer to the initial level after over 6 months of observation. During the follow-up period, the average increase in CT was the highest in the M-region (7.54 \pm 5.51 $\mu \mathrm{m})$ and the mean CT was the thickest in the M-region $(267.46 \pm 52.70 \mu \mathrm{m})$ at 3 months after orthokeratology lens wearing. The thinnest choroid was at $\mathrm{N}$-region, which was significantly thinner than that at the M-region ( $p=0.011$, Tukey test). The interobserver intraclass coefficients of the 2 observers ranged from 0.931 to 0.997 . The mean absolute errors of the 2 observers were calculated as $<6.8 \mu \mathrm{m}$. The Box plot was prepared to show mean CT measured before and after orthokeratology treatment as a quartile (Fig. 3). 
Fig. 2. Longitudinal changes of mean CT before and after orthokeratology treatment. CT is significantly increased at 3 months after orthokeratology treatment. After that, it is inclined to go back to baseline. Central, subfoveal region; T1500, temporal 1,500 $\mu \mathrm{m}$; N1500, nasal 1,500 $\mu \mathrm{m}$; CT, choroidal thickness.

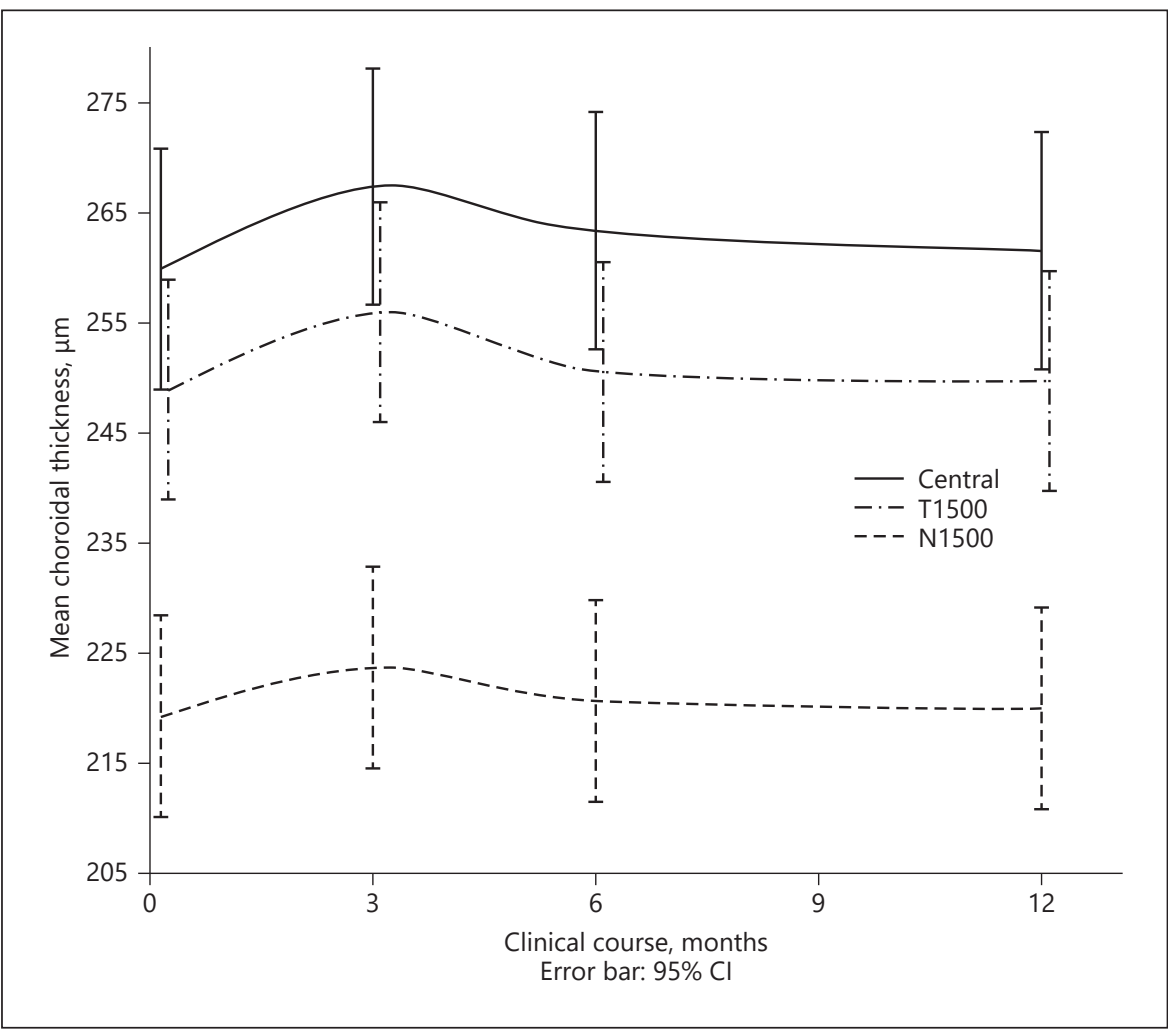

Table 2. Mean CT of horizontal axis before and after orthokeratology treatment $(\mu \mathrm{m})$

\begin{tabular}{|c|c|c|c|c|}
\hline Time & N1500 & Central & T1500 & p value ${ }^{b}$ \\
\hline Baseline & $219.23 \pm 46.46$ & $259.92 \pm 55.33$ & $248.96 \pm 45.72$ & $0.012^{\mathrm{a}}$ \\
\hline 3 months & $223.62 \pm 47.31$ & $267.46 \pm 53.74$ & $255.92 \pm 46.88$ & $0.006^{\mathrm{a}}$ \\
\hline$p$ value $^{c}$ & $<0.01^{\mathrm{a}}$ & $<0.01^{\mathrm{a}}$ & $<0.01^{\mathrm{a}}$ & \\
\hline 6 months & $220.65 \pm 46.82$ & $263.35 \pm 54.79$ & $250.54 \pm 45.31$ & $0.008^{\mathrm{a}}$ \\
\hline$p$ value ${ }^{c}$ & $0.039^{\mathrm{a}}$ & $<0.01^{\mathrm{a}}$ & 0.220 & \\
\hline 12 months & $219.96 \pm 47.15$ & $261.81 \pm 51.68$ & $249.73 \pm 45.14$ & $0.009^{\mathrm{a}}$ \\
\hline$p$ value $^{c}$ & 0.095 & $<0.01^{\mathrm{a}}$ & 0.472 & \\
\hline
\end{tabular}

Values are presented as mean $\pm \mathrm{SD}$, unless otherwise indicated. $\mathrm{CT}$, choroidal thickness; central, subfoveal region; T1500, temporal $1,500 \mu \mathrm{m}$; N1500, nasal $1,500 \mu \mathrm{m}$. ${ }^{\mathrm{a}}$ Indicates statistical significance. ${ }^{\mathrm{b}}$ Paired $t$ test (comparing CT, before and after orthokeratology treatment). ${ }^{c}$ Tukey test (comparing among groups).

\section{Discussion/Conclusion}

The choroid is a vascular structure between the retina and the sclera. It supplies retinal pigment epithelium and outer retina. It has several functions, including oxygenation, nourishment, thermoregulation of outer retinal layers, adjustment of the retinal position by changes in $\mathrm{CT}$, and secretion of growth factors [7]. OCT technology is now widely used in the diagnosis and monitoring of posterior retinal and choroidal pathology in humans [21]. An improved understanding of characteristics of normal thicknesses of the retina and choroid should assist in the diagnosis of abnormalities in these structures known to be associated with ocular diseases [21]. CT may vary depending on various conditions [21-24]. Increasing age has been shown to be significantly correlated with de- 
Fig. 3. Box plot showing difference between $\mathrm{CT}$ and each region before and after orthokeratology treatment. N1500, nasal 1,500 $\mu \mathrm{m}$; central, subfoveal region; T1500, temporal 1,500 $\mu \mathrm{m}$; CT, choroidal thickness.

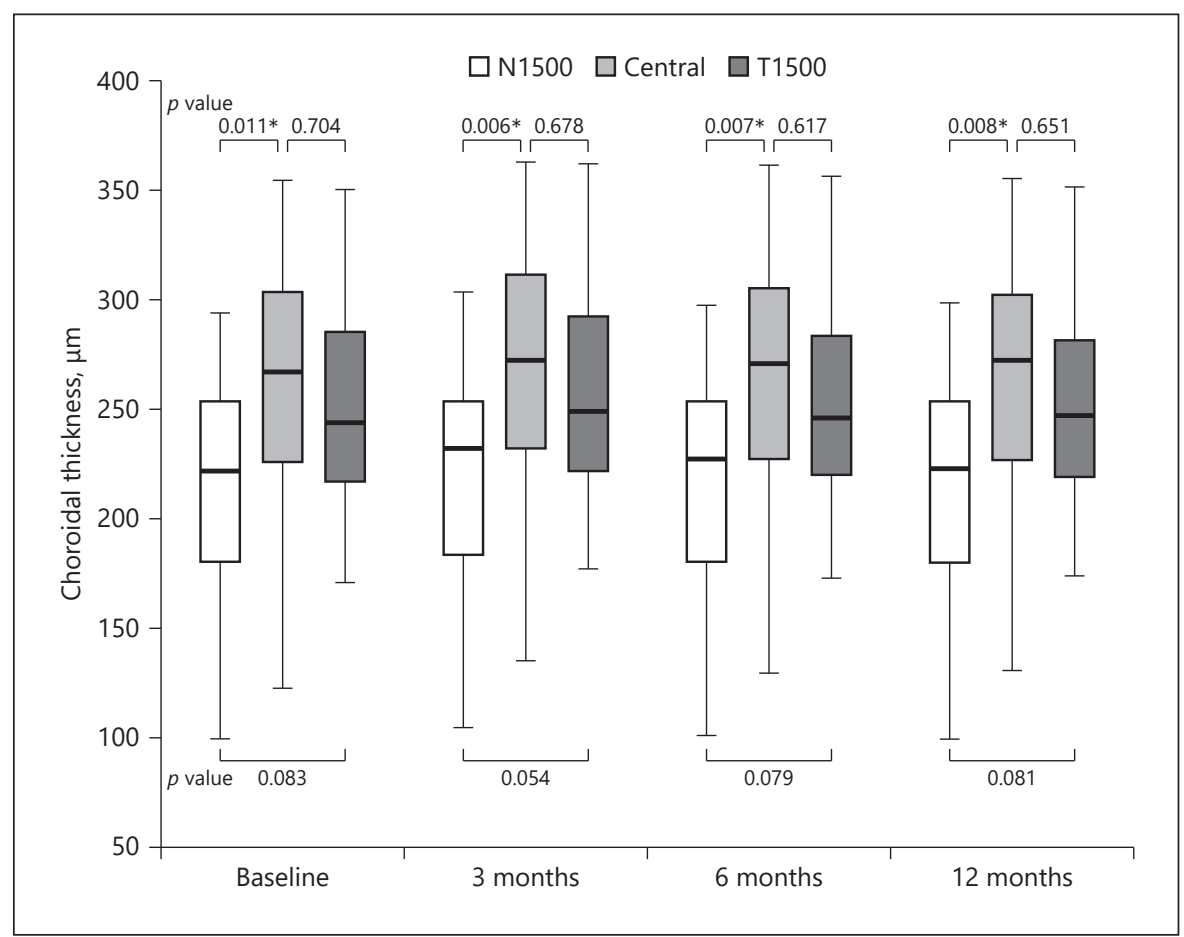

creasing CT [25-27]. CT undergoes significant diurnal variation (mean change, $0.029 \pm 0.016 \mathrm{~mm}$ ). It is typically thicker at night and thinnest in the morning [28]. Some studies have suggested that gender and hormonal status may influence choroidal blood flow and lead to change in CT $[29,30]$.

We conducted a study on young myopic adult patients who did not want glasses or refractive surgery but wanted orthokeratology treatment. The average CT was the thickest underneath the fovea $(259.92 \pm 55.33 \mu \mathrm{m})$. The mean CT of temporal region was thicker than the $\mathrm{N}$-region. This regional thickness difference of choroid was consistent with other studies [26, 27, 31, 32]. However, baseline values before orthokeratology treatment in our study were somewhat different from those reported previously by others. Such differences might have resulted from differences in measuring software or the OCT device, differences in ethnicity, or differences in patient profiles such as age, refractive errors, and axial length [27]. Ding et al. [27] have reported a subfoveal CT of $293.51 \mu \mathrm{m}$ in adults aged $20-29$ years. Yasushi et al. [26] have reported a mean CT of $354 \pm 111 \mu \mathrm{m}$ at the fovea.

The cause of increase in CT after orthokeratology treatment in myopic young adults is unknown. We have several hypotheses. First, the increase in CT might have increased choroidal vascular circulation due to increased visual function with signals that could inhibit myopia by peripheral defocus. The returning of CT to the baseline value at 6 months after orthokeratology treatment might control choroidal homeostasis in ocular function. The underlying physiological mechanism remains unclear. After theoretically successful orthokeratology treatment, the fovea becomes emmetropic and the peripheral retinal zone becomes myopic defocus. Induced peripheral myopic defocus might push the retina forward and make the choroid thicker. Our understanding of refractive error development has been greatly enhanced by lens compensation experiments [12]. Experiments in several animal species have shown that myopic defocus can slow axial growth of the eye $[11,33,34]$. The second reason for the increase of CT might be due to negative hydraulic pressure between the cornea and the orthokeratology lens caused by overnight lens wearing. The reverse geometry lens design increases viscosity of trapped tears overnight, creating a negative hydraulic pressure and suction cup effect in the morning $[35,36]$. This phenomenon might have affected homeostatic control of the choroid and led to thickening of the choroid.

This study has several limitations. First, the sample size was relatively small. In addition, age range and refractive error of subjects were limited. Second, subjects 
may not have regularly worn orthokeratology lenses after obtaining satisfactory vision during the follow-up period. This is because after a certain period of stabilization between 1 and 2 months of beginning orthokeratology treatment, some subjects may get satisfactory vision even if they do not wear orthokeratology lenses every day.

Observers used only subfoveal scanned choroidal image with central locations without retinal tilt for inter- or intraobserver repeatability. To reduce measurement error, stromal CT was estimated. The 2 observers agree on how to demarcate the choroidal boundary before the study. Last, OCT imaging of all patients were examined every Friday morning (09:30 a.m.-12:30 p.m.). Therefore, influence of the diurnal variation would be limited.

Young myopic Korean adults with an average age of $23.6 \pm 2.5$ years showed increase in mean CT during the first 3 months after orthokeratology treatment. Thus, mean CT can be used as an important parameter for examining choroids in young myopic patients with ocular disease receiving orthokeratology treatment.

\section{Statement of Ethics}

This study was conducted in accordance with the tenets of the Declaration of Helsinki and approval from the Institutional Review Board/Ethics Committee of the Dongtan Sacred Heart Hospital (IRB No. HDT 2020-05-018). Informed consent was obtained in writing from all participants after explaining the nature of orthokeratology.

\section{Conflict of Interest Statement}

The authors have no conflicts of interest to declare.

\section{Funding Sources}

Grant: None. Funding/Support: None. Financial Disclosures: The authors have no financial disclosures to report.

\section{Author Contributions}

Study design and concept: J.H.L. and G.S.J.; database search: J.H.L., G.S.J., and I.H.H.; data extracting: T.Y.L., J.H.L., and I.H.H.; data analysis: J.H.L., I.H.H., and T.Y.L.; manuscript writing: J.H.L., G.S.J., and I.H.H.; manuscript revising J.H.L., G.S.J., and J.R.H. All authors approved the final version of the manuscript.

\section{References}

1 Walline JJ, Jones LA, Sinnott LT. Corneal reshaping and myopia progression. $\mathrm{Br} \mathrm{J} \mathrm{Oph}-$ thalmol. 2009 Sep;93(9):1181-5.

2 Swarbrick HA, Alharbi A, Watt K, Lum E, Kang P. Myopia control during orthokeratology lens wear in children using a novel study design. Ophthalmology. 2015 Mar; 122(3): 620-30.

3 Si JK, Tang K, Bi HS, Guo DD, Guo JG, Wang XR. Orthokeratology for myopia control: a meta-analysis. Optom Vis Sci. 2015 Mar; 92(3):252-7.

4 Kakita T, Hiraoka T, Oshika T. Influence of overnight orthokeratology on axial elongation in childhood myopia. Invest Ophthalmol Vis Sci. 2011 Apr 6;52(5):2170-4.

5 Li Z, Cui D, Hu Y, Ao S, Zeng J, Yang X. Choroidal thickness and axial length changes in myopic children treated with orthokeratology. Cont Lens Anterior Eye. 2017 Dec;40(6): 417-23.

6 Kang P, Swarbrick H. Peripheral refraction in myopic children wearing orthokeratology and gas-permeable lenses. Optom Vis Sci. 2011 Apr;88(4):476-82.

7 Nickla DL, Wallman J. The multifunctional choroid. Prog Retin Eye Res. 2010 Mar;29(2): 144-68.
8 Chen Z, Xue F, Zhou J, Qu X, Zhou X. Effects of orthokeratology on choroidal thickness and axial length. Optom Vis Sci. 2016 Sep; 93(9):1064-71.

9 Hung LF, Wallman J, Smith EL 3rd. Visiondependent changes in the choroidal thickness of macaque monkeys. Invest Ophthalmol Vis Sci. 2000 May;41(6):1259-69.

10 Troilo D, Nickla DL, Wildsoet CF. Choroidal thickness changes during altered eye growth and refractive state in a primate. Invest Ophthalmol Vis Sci. 2000 May;41(6):1249-58.

11 Wildsoet C, Wallman J. Choroidal and scleral mechanisms of compensation for spectacle lenses in chicks. Vision Res. 1995 May;35(9): 1175-94.

12 Gardner DJ, Walline JJ, Mutti DO. Choroidal thickness and peripheral myopic defocus during orthokeratology. Optom Vis Sci. 2015 May;92(5):579-88.

13 Chen Z, Xue F, Zhou J, Qu X, Zhou X. Effects of orthokeratology on choroidal thickness and axial length. Optom Vis Sci. 2016 Sep; 93(9):1064-71.

14 Li Z, Cui D, Hu Y, Ao S, Zeng J, Yang X. Choroidal thickness and axial length changes in myopic children treated with orthokeratology. Cont Lens Anterior Eye. 2017 Dec;40(6): 417-23.
15 Jin WQ, Huang SH, Jiang J, Mao XJ, Shen MX, Lian Y. Short term effect of choroid thickness in the horizontal meridian detected by spectral domain optical coherence tomography in myopic children after orthokeratology. Int J Ophthalmol. 2018 Jun 18;11(6): 991-6.

16 Gonzalez-Meijome JM, Villa-Collar C. Nomogram, corneal topography, and final prescription relations for corneal refractive therapy. Optom Vis Sci. 2007 Jan;84(1):59-64.

17 Queirós A, González-Méijome JM, Villa-Collar C, Gutiérrez AR, Jorge J. Local steepening in peripheral corneal curvature after corneal refractive therapy and LASIK. Optom Vis Sci. 2010 Jun;87(6):432-9.

18 Kiel JW, van Heuven WA. Ocular perfusion pressure and choroidal blood flow in the rabbit. Invest Ophthalmol Vis Sci. 1995 Mar; 36(3):579-85.

19 Regatieri CV, Branchini L, Fujimoto JG, Duker JS. Choroidal imaging using spectral-domain optical coherence tomography. Retina. 2012 May;32(5):865-76.

20 Shao L, Xu L, Wei WB, Chen CX, Du KF, Li $\mathrm{XP}$, et al. Visual acuity and subfoveal choroidal thickness: the Beijing Eye Study. Am J Ophthalmol. 2014 Oct;158(4):702-9.e1. 
21 Li T, Zhou X, Wang Z, Zhu J, Shen W, Jiang B. Assessment of retinal and choroidal measurements in Chinese school-age children with cirrus-HD optical coherence tomography. PLoS One. 2016 Jul 8;11(7):e0158948.

22 Faghihi H, Hajizadeh F, Riazi-Esfahani M. Optical coherence tomographic findings in highly myopic eyes. J Ophthalmic Vis Res. 2010 Apr;5(2):110-21.

23 Yeoh J, Rahman W, Chen F, Hooper C, Patel $\mathrm{P}$, Tufail A, et al. Choroidal imaging in inherited retinal disease using the technique of enhanced depth imaging optical coherence tomography. Graefes Arch Clin Exp Ophthalmol. 2010 Dec;248(12):1719-28.

24 Zhou M, Wang W, Ding X, Huang W, Chen $\mathrm{S}$, Laties AM, et al. Choroidal thickness in fellow eyes of patients with acute primary angleclosure measured by enhanced depth imaging spectral-domain optical coherence tomography. Invest Ophthalmol Vis Sci. 2013 Mar 19; 54(3):1971-8.

25 Margolis R, Spaide RF. A pilot study of enhanced depth imaging optical coherence tomography of the choroid in normal eyes. Am J Ophthalmol. 2009 May;147(5):811-5.
26 Ikuno Y, Kawaguchi K, Nouchi T, Yasuno Y. Choroidal thickness in healthy Japanese subjects. Invest Ophthalmol Vis Sci. 2010 Apr; 51(4):2173-6.

27 Ding X, Li J, Zeng J, Ma W, Liu R, Li T, et al. Choroidal thickness in healthy Chinese subjects. Invest Ophthalmol Vis Sci. 2011 Dec 20; 52(13):9555-60.

28 Chakraborty R, Read SA, Collins MJ. Diurnal variations in axial length, choroidal thickness, intraocular pressure, and ocular biometrics. Invest Ophthalmol Vis Sci. 2011 Jul 11;52(8): 5121-9.

29 Kavroulaki D, Gugleta K, Kochkorov A, Katamay R, Flammer J, Orgul S. Influence of gender and menopausal status on peripheral and choroidal circulation. Acta Ophthalmol. 2010 Dec;88(8):850-3.

30 Centofanti M, Bonini S, Manni G, GuinettiNeuschüler C, Bucci MG, Harris A. Do sex and hormonal status influence choroidal circulation? Br J Ophthalmol. 2000 Jul;84(7): 786-7.
31 Read SA, Collins MJ, Vincent SJ, Alonso-Caneiro D. Choroidal thickness in myopic and nonmyopic children assessed with enhanced depth imaging optical coherence tomography. Invest Ophthalmol Vis Sci. 2013 Nov 15; 54(12):7578-86.

32 Read SA, Collins MJ, Vincent SJ, Alonso-Caneiro D. Choroidal thickness in childhood. Invest Ophthalmol Vis Sci. 2013 May 1;54(5): 3586-93.

33 Howlett MH, McFadden SA. Spectacle lens compensation in the pigmented guinea pig. Vision Res. 2009 Jan;49(2):219-27.

34 Smith EL 3rd, Hung LF, Huang J. Relative peripheral hyperopic defocus alters central refractive development in infant monkeys. Vision Res. 2009 Sep;49(19):2386-92.

35 Stillitano I, Maidana E, Lui M, Lipener C, Höfling-Lima AL. Bubble and corneal dimple formation after the first overnight wear of an orthokeratology lens: a case series. Eye Contact Lens. 2007 Sep;33(5):253-8.

36 Lin ST, Leahy CD, Mandell RB. The effect of time, patching, and lens flexibility on RGP lens adherence. J Am Optom Assoc. 1989 Mar;60(3):182-7. 